\title{
Electronically-switched Directional Antennas for Wireless Sensor Networks: A Full-stack Evaluation
}

\author{
Luca Mottola $^{\dagger \ddagger}$, Thiemo Voigt ${ }^{+\ddagger}$, Gian Pietro Picco* \\ $\dagger$ Politecnico di Milano (Italy), $\ddagger$ SICS (Sweden), \\ +Uppsala University (Sweden), *University of Trento (Italy)
}

\begin{abstract}
We study the benefits of electronically-switched directional (ESD) antennas in wireless sensor networks (WSNs). ESD antennas have proved beneficial in cellular and ad-hoc networks, by increasing the communication range and by alleviating contention in directions other than the destination. The advantages in WSNs are, however, still largely to be quantified. Unlike existing works in this field, we start by characterizing a real-world antenna prototype, and apply this to an existing WSN stack, which we adapt with minimal changes. Our results show that: $i$ ) the combination of a low-cost ESD antenna and a mainstream WSN stack already brings significant performance improvements, e.g., nearly halving the radio-on time per delivered packet; ii) the margin of improvement available to alternative clean-slate protocol designs is similarly large and concentrated in the control rather than the data plane; iii) by artificially modifying our antenna's link-layer model, further potential benefits opened by different antenna designs may be available. To the best of our knowledge, this is the first study providing such quantitative insights based on a real ESD antenna prototype and a complete WSN stack.
\end{abstract}

\section{INTRODUCTION}

Electronically-switched directional (ESD) antennas are capable of dynamically conveying the radiated power along given directions, unlike the isotropic propagation of omni-directional ones. Therefore, they can alleviate wireless contention by not involving nodes other than the destination, and increase communication range essentially at no additional energy cost.

These benefits are demonstrated in the vast existing literature on cellular and ad-hoc networks. However, ESD antennas are particularly appealing for low-power wireless sensor networks (WSNs), where energy consumption is key. Unfortunately, the related literature for WSNs is significantly more sparse. Moreover, as we discuss in Section II, most of the few works available: $i$ ) focus on a single component of the network stack, or ii) rely on simulations based on ideal radio models. These aspects prevent an immediate use of these results in WSN deployments, as the latter require a full stacks and real antennas. Further, and more importantly, they leave unanswered the fundamental question of whether and to what extent ESD antennas practically improve WSN performance.

This paper addresses this question by adopting a different perspective, which sets us apart from the related literature:

1) instead of proposing clean-slate protocols for ESD antennas, we start from a mainstream WSN stack and adapt it with the minimal changes necessary to exploit directional transmissions. In a sense, instead of aiming for the maximum benefits ESD antennas may yield through a clean-slate design, we focus on the minimum ones that can be harvested from protocols that have been widely applied, understood, and improved [14], [15].

2) instead of relying on idealized antenna models in our evaluation, we use an empirical model built from realworld experiments on a prototype of ESD antenna, expressly designed for integration with WSN devices. In a sense, we trade the generality of ideal models for the practical relevance of results based on a real prototype.

The prototype we use is the SPIDA antenna by Nilsson [19], whose features we summarize in Section III along with the empirical link-layer model we derive for it. The latter allows us to analyze through simulation large-scale settings that would be otherwise difficult to reproduce in a real deployment, due to limited availability of antenna prototypes.

The mainstream WSN stack we adapt is Collect [15], the reference tree-based collection protocol in the Contiki operating system. We perform minimal modifications to it to support directional packet forwarding (DPF) from a node to its parent in the tree. The rest of the routing protocol (e.g., tree maintenance) and of the stack (e.g., MAC) is unaltered, and relies on omni-directional communication. We illustrate the details of the protocols in Section IV.

We compare our modified Collect against two baselines. The first one is the original Collect, with the antennas in omni-directional mode. This represents the lower bound our modified Collect improves upon. The latter stack, however, still builds the routing tree through omni-directional communication, and does not fully exploit the benefits of ESD antennas. The increased range of the latter may indeed yield fewer hops to reach the destination, reducing the number of transmissions.

Therefore, our second baseline is a DPF schema operating on an optimal topology, computed offline by taking into account the increased communication range of SPIDA. Section V describes the analytical formulation of the corresponding routing problem and its optimal solution. This second baseline represents the upper bound for the improvement brought by the modified Collect we consider. The performance gap of the modified Collect to this second baseline represents the margin of improvement available to clean-slate protocol designs.

Our evaluation methodology is described in Section VI, 
whereas we present experimental results in Section VII. The main findings, constituting the core contribution of the paper, are that: $i$ ) the combination of a low-cost directional antenna and a conventional network stack already brings significant performance improvements, e.g., nearly halving the radio-on time per delivered packet; ii) the margin of improvement available to clean-slate protocol designs is similarly large and concentrated in the control rather than the data plane; iii) by artificially altering our link-layer model to investigate antennas with different characteristics, we point at additional potential benefits available to alternative antenna designs.

Section VIII ends the paper with brief concluding remarks.

\section{RELATED WORK}

ESD antennas-as well as other forms of dynamically steerable directional transmissions, such as switched and adaptive beamforming - are widely employed in cellular and ad-hoc networks. A significant body of work exists in this field [7]. Examples of the most recent developments are the work by Arslan et al. [1], who design an efficient Wi-Max beamformer antenna and implement real prototypes, and several works on adaptive beamforming for indoor wireless LANs [5] that include real-world validations.

We need to play on a different ground, as the solutions designed for cellular and ad-hoc wireless networks can hardly find their way in WSNs [2]. The key performance metrics are different: the former types of networks focus mostly on throughput and latency, whereas in WSNs packet delivery and energy consumption are paramount. The traffic patterns and network topologies are also incompatible: cellular and adhoc networks tend to show one-to-one or one-to-many traffic in mostly star-shaped topologies. In WSNs, traffic is largely many-to-one across an unstructured multi-hop topology.

The literature investigating the use of ESD antennas in WSNs can be roughly divided in two categories, focusing either: i) on the design of antenna prototypes; or ii) on clean-slate design of network protocols. Works in $i$ ) were instrumental in concretely demonstrating the viability of ESD antennas in WSNs, showing that current technology can meet the requirements on form factor and energy consumption. However, these works rarely assess the impact of the prototypes on the network stack. Specifically, Giorgetti et al. [12] assess the improvements in link performance with a prototype designed by combining four patch antennas. Viani et al. [24] present a design based on parasitic elements, and analyze its ability to reduce interference and to support localization. Parasitic elements are a common choice to reduce cost and size [5] used also by Nilsson [19] in the SPIDA design, later assessed based on common metrics for WSNs [20].

In the second category, protocols are often designed based on idealized antenna models defined purely by geometrical properties. For example, Felemban et al. [9] propose a cleanslate protocol stack that relies solely on directional communications, emphasizing neighbor discovery based on their previous work [10]. Although their stack may, in principle, be implemented atop ESD antennas, their simulation results

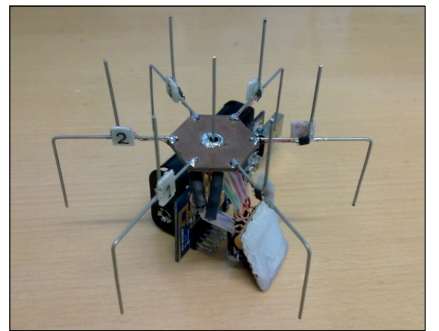

Fig. 1. SPIDA prototype, connected to a TMote Sky node.

do not translate immediately to a real antenna. Other works focus on specific network services considered in isolation, e.g., neighbor discovery [23] and MAC [9], only seldom including real-world validations.

\section{Antenna Prototype AND LinK-LAyer Model}

We use a real-world ESD antenna prototype to derive an empirical link-layer model. In Section VII, we use this model to assess the impact of this antenna technology in WSNs.

\section{A. Antenna Prototype}

Most radio chips for WSNs operate in the $2.4 \mathrm{GHz}$ ISM band. This, together with the requirement of small form factor limits the choice of antenna technology in this context [19].

We use a switched parasitic element antenna [22] called SPIDA, designed by Nilsson [19]. It consists of a central active element surrounded by "parasitic" elements, as in Figure 1. The central element is a quarter-wavelength whip antenna, i.e., a traditional omni-directional antenna. The parasitic elements can be switched between ground and isolation: when grounded, they work as reflectors of radiated power; when isolated, they act as directors of radiated power. SPIDA has six parasitic elements, yielding six possible "switches" to control the shape and direction of the antenna main lobe. The parasitic elements are individually controllable: when all isolated, SPIDA behaves as an omni-directional antenna, which simplifies broadcasting and neighbor discovery.

The antenna gain is designed to smoothly vary as an offset circle from approximately $7 \mathrm{~dB}$ to $-4 \mathrm{~dB}$ in the horizontal plane, with the highest gain in the direction of the isolated element(s). The antenna is also straightforward to manufacture, and its most expensive part is the SMA connector (about $\$ 6$ in single quantities). The cost, size, and radiation characteristics of SPIDA are therefore comparable with the state of the art in directional antennas for WSNs [5], [12], [24], rendering our results of general applicability.

\section{B. Link-layer Model}

Modeling. We hook our SPIDA prototype to a TMote Sky node. We place the node in an open grass field, surrounded by 16 additional TMote Sky nodes with standard omni-directional antennas, deployed in a grid configuration. These act as probes by logging packets received from the SPIDA node, which broadcasts batches of 1000 packets of varying sizes, from 20 bytes to 100 bytes, with an inter-packet interval (IPI) of $500 \mathrm{~ms}$. We perform different experiments with different values of environmental temperature and humidity. Overall, 


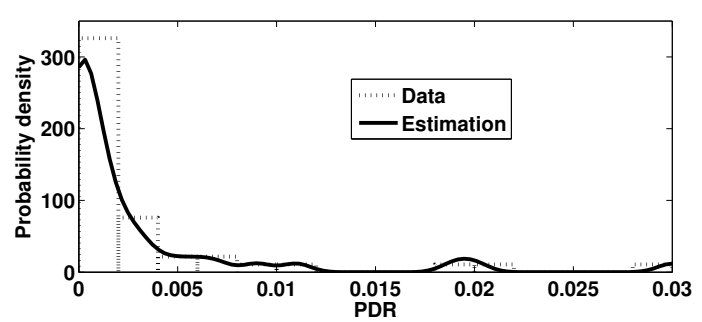

(a) Probe outside SPIDA main lobe with low PDR.

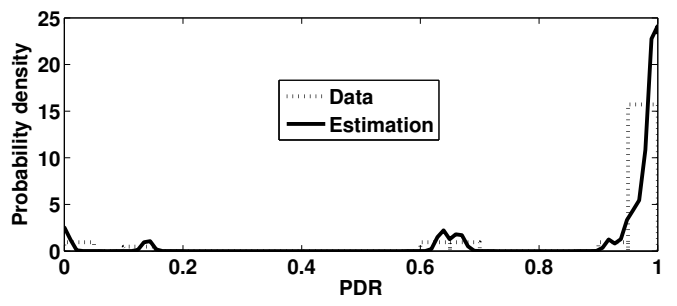

(b) Probe inside SPIDA main lobe with high $P D R$.

Fig. 2. PDF at different probe nodes.

the probes collect more than a million packets. We illustrate further details on the setup in an extended report [17].

Based on these data, we derive a spatio-temporal model of Packet Delivery Rate (PDR) and Received Signal Strength Indicator (RSSI) for SPIDA, borrowing from Cerpa et al. [4]. In contrast to their work, however, our model also considers the directionality of the antenna. This requires two independent variables describing coordinates in a plane, rather than only distance from the source. We model the average $P D R$ and $R S S I$ measured by a probe at the end of every batch as instances of a random variable. We then apply kernel density estimation to identify the corresponding probability density function (PDF). This method is particularly accurate in absence of information on the underlying probability distribution. The data we obtain grants a $95 \%$ confidence interval.

To exemplify the results, Figure 2 depicts two example PDFs for $P D R$, corresponding to different probes. Figure 2(a) describes a probe outside the main antenna lobe. The PDF shows a single maximum for low values of $P D R$, although some packets are still occasionally received. We show in Figure 2(b) the PDF for PDR at a probe in the middle of the main antenna lobe: the situation is opposite, as the PDF shows a maximum for high values of $P D R$. In the resulting PDFs we often observe such bi-modal behavior, where packets are either received with very high probability, or only seldom received. This makes the PDFs also largely independent of packet sizes, as the behavior is mainly determined by the receiver location.

By discretizing the PDF curves, we generate probability tables that associate given probability densities with specific values of $P D R$ and $R S S I$. We use these tables to obtain the corresponding empirical cumulative distribution functions (ECDFs). Based on these, we apply inverse transform sampling to generate new random values with the same statistical trends as the original data. In essence, these model the timevarying nature of wireless links when using SPIDA to transmit.

Figure 3 shows the region of space with average $P D R>10 \%$ obtained from the empirical model, with SPIDA

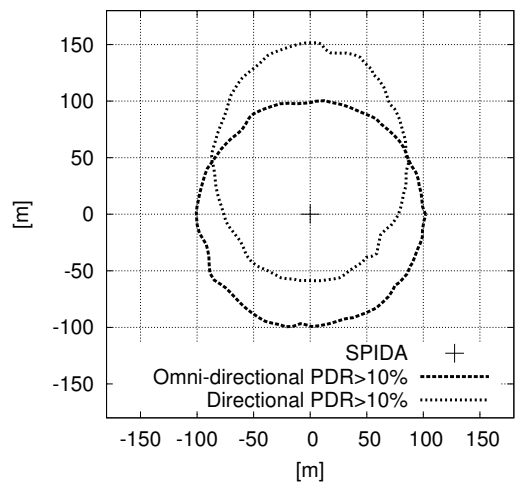

Fig. 3. Region of space with $P D R>10 \%$., from the empirical model.

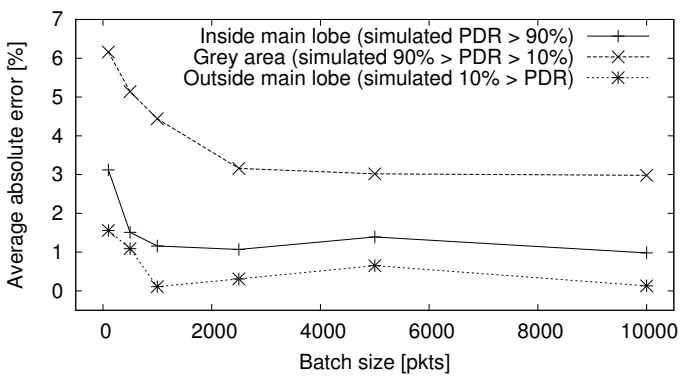

(a) Simulation and real experiments vs. different packet batches.

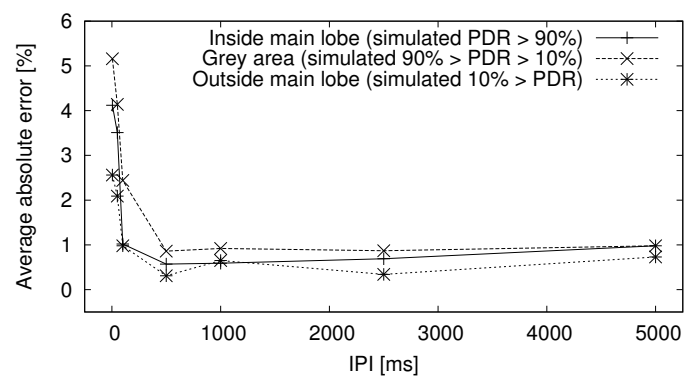

(b) Simulation and real experiments vs. different IPIs.

Fig. 4. Average absolute error simulation vs. real experiments.

isolating only the element aligned with the vertical axis pointing upwards. Compared to the omni-directional setting, the area with $P D R>10 \%$ becomes slightly narrower and is offset in the direction of the isolated element, verifying the original design [19].

Validation. We validate our model to assess: $i$ ) the extent the model adheres to the real-world behavior beyond the experimental data it is derived from; and ii) how general is our choice of batch size and IPI during the original experiments.

We gather further real-world data we compare with data generated by the implementation of our model in the Cooja/MSPSim [8] simulator, using an emulated CC2420 radio chip. The validation data is gathered at the same place as the original data, but with a random placement of the probes and under very different temperature and humidity conditions. We let the SPIDA node send another one million packets at different IPIs. We replicate the same setup and positioning in Cooja and simulate the experiment. We repeat this about 50 times by varying positions of the probes. We then split the data in batches of different sizes.

Figure 4 compares the $P D R$ obtained in simulation against the real experiment. The results show that our model is 
accurate also against the validation data. The worst case error is slightly above $6 \%$ and holds for nodes with neither very strong or very weak links, as the statistical variability is higher for this kind of links [21]. Moreover, the error is generally higher for smaller packet batches and smaller IPIs. This is expected because: $i$ ) when the IPI is sufficiently small, packet losses become dependent [21]; and ii) with too few packets, the results are no longer statistically significant.

\section{PRotocols}

We describe the protocols we consider to understand the immediate benefits of ESD antennas in WSNs with the changes we apply to enable directional packet forwarding (DPF).

Base protocol stack. We define an OMNI protocol configuration that exclusively uses omni-directional transmissions. We use Contiki's default tree-based collection protocol, Collect [15], similar to the Collection Tree Protocol (CTP) [13] in the TinyOS distribution. Several real-world deployments are based on these protocols or variations thereof [11].

Like most such protocols, Collect includes two core functionality: $i)$ building and maintaining multi-hop routes in a tree-shaped topology; and ii) forwarding application data up the routing tree. For $i$ ), Collect relies on ETX [6] as a routing metric. ETX measures the number of 1-hop transmissions to deliver a packet until receiving an explicit acknowledgment. Collect builds routes to minimize the total ETX from sources to the data sink. Although in newer Contiki versions the ETX is estimated using explicit unicast messages [15], in our version nodes broadcast these advertisements as in CTP.

We use Contiki's default low-power MAC protocol, ContikiMAC. Similar to X-MAC [3], senders transmit small probe packets containing the receiver's identifier until the latter wakes up and acknowledges the strobe packet, whereupon the sender transmits the data packet.

Directional packet forwarding (DPF). Our goal is to explore the advantages we can readily harvest with ESD antennas. Thus, we aim at keeping the changes to the OMNI stack at a minimum: to leverage directional transmissions, we modify only the data forwarding to the tree parent. Exploiting the directional transmissions also for tree building is considerably more complex, as discussed in Section VI. Moreover, the smaller the changes, the more ESD antennas may leverage the existing networking codebase and understanding of current protocols [14]. We devise three simple schemes:

- BLIND forwarding entails quickly transmitting every packet in all six SPIDA directions, in sequence. As the packet remains in the radio buffer while switching direction, subsequent transmissions still happen within the original radio activation times of ContikiMAC, which include sufficient guard times. No support from hardware acknowledgements is needed, either. Implementing BLIND thus requires only 4 lines of $\mathrm{C}$ code in Collect.

- NARROW forwarding randomly selects only one direction. Should the receiver send an acknowledgement back, the sender continues sending using the same direction, until either an acknowledgment is lost or the parent changes. When so, it re-starts from another random direction. NARROW requires changes to 12 lines of code.

- SMART forwarding searches for a working configuration by quickly sweeping all directions, as in BLIND. However, to detect which direction succeeded, it appends the identifier of the active one to the packet, encoded with a corresponding number of padding bytes. Packets can therefore be transmitted without re-loading the radio buffer, since only the total packet size is changed, but not the content. SMART requires changes to 16 lines of code.

In all these schemes, the MAC protocol is thus unaltered, including the strobing occurring in omni-directional mode, and the changes are restricted to the child side: packet receptions and unicast acknowledgments of application data always occur with SPIDA in omni-directional mode.

\section{Routing Problem and Optimal Solution}

We formulate the routing problem with ESD antennas as an integer linear program (ILP), which we use later to compute the optimal configuration of the routing tree. Our approach builds upon the multi-commodity problem [25], a formulation already applied in wireless networks [18]

In a multi-commodity routing problem, we consider a directed graph, e.g., representing a transportation network, with node set $\mathcal{N}$ and arc set $\mathcal{A}$, and a set of commodities $\mathcal{C}$, e.g. goods. The goal is to route each commodity $k \in \mathcal{C}$ from a set of origins $O(k) \subseteq \mathcal{N}$ to a set of destinations $D(k) \subseteq \mathcal{N}$ by minimizing a given metric.

System model. We model a wireless network as the directed graph where $\mathcal{N}$ is composed of the WSN nodes, commodities in $\mathcal{C}$ are packets flowing from sources to destinations, and $\mathcal{A}$ contains an arc $(i, j)$ if a packet sent by node $i$ has nonzero probability of being received at node $j$. Unlike existing works [18], we do not model this notion of communication with a Boolean value indicating whether communication is possible. Instead, each link has an associated weight $w_{i, j}$ representing the quality of the link based on its $P D R$.

We derive $P D R$ information between any two nodes with our empirical link-layer model. During dedicated simulations, a simple discovery protocol instructs each node $i$ to send 10,000 broadcast messages for each direction the SPIDA allows by isolating a single parasitic element (i.e., 60,000 messages in total). All other nodes $j \log$ the messages received. If communication between two nodes is possible through different directions, the highest $P D R$ is assigned as a weight to the link and the corresponding antenna configuration recorded with the link $(i, j)$. Thus, we do not explicitly model the different SPIDA directions, rather we associate them to links, simplifying the modeling.

In our context, the destinations $D(k)$ always consist of a single node, the tree root $d$. Without loss of generality, we assume a commodity to flow from a single origin to the destination [25]. Since commodities flowing from the same origin to the destination follow the same route, we can state a one-to-one mapping between the route connecting any pair $\langle o(k), d\rangle$, and any commodity $k$. 
We model the fact that a link from $i$ to $j$ is used for routing from a given source $k$ to the tree root $d$ by using Boolean variables attached to each link:

$$
r_{i, j}^{k}= \begin{cases}1 & \text { if route from source } k \text { contains link }(i, j) \\ 0 & \text { otherwise }\end{cases}
$$

Objective function. Collection protocols for WSNs often rely on ETX to build end-to-end estimates of a routing path's quality. To include ETX information in our modeling, given the PDR information for links $(i, j)$ and $(j, i)$, we first compute the probability that a packet is sent $n$ times on $(i, j)$ before an acknowledgment is received on $(j, i)$ :

$P(R T X=n)_{i, j}=\left(1-P D R_{i, j} P D R_{j, i}\right)^{(n-1)} P D R_{i, j} P D R_{j, i}$

Let the probability of a successful bidirectional transmission be $P_{T X}=P D R_{i, j} P D R_{j, i}$. The expected number of transmissions $R T X$ from $i$ to $j$, i.e., what ETX tries to measure, is $E(R T X)_{i, j}=\frac{1}{P_{T X}}$. Thus, the cost of a link from $i$ to $j$ in a route from source $k$ is:

$$
u_{i, j}^{k}= \begin{cases}E(R T X)_{i, j}=\frac{1}{P_{T X}} & \text { if } r_{i, j}^{k}=1 \\ 0 & \text { otherwise }\end{cases}
$$

The goal is then to minimize the overall expected number of transmissions for all links used by all routes, that is:

$$
\operatorname{Tree} \operatorname{Cost}(\mathcal{C}, \mathcal{A})=\sum_{k \in \mathcal{C},(i, j) \in \mathcal{A}} u_{i, j}^{k}
$$

Optimal solution. We are to find the value assignment of $r_{i, j}^{k}, \forall k \in \mathcal{C}, \forall(i, j) \in \mathcal{A}$ such that $\operatorname{Tree} \operatorname{Cost}(\mathcal{C}, \mathcal{A})$ is minimum. We derive the optimal solution to the problem using mathematical programming, which requires specifying the necessary constraints on the decision variables.

First, we constrain $r_{i, j}^{k}$ to be an integer binary variable. Second, we state that the assignment to $r_{i, j}^{k}$ must contain a connected end-to-end path for each route $k$. This constraint can be expressed by requiring every node different from source $o(k)$ and destination $d$ to "preserve" packets. In other words, if a route "enters" node $i$, it must also exit from it, unless it is a source or the destination:

$$
\begin{aligned}
& \forall i \in \mathcal{N}, \forall k \in \mathcal{C}, \\
& \sum_{m:(i, m) \in \mathcal{A}} r_{i, m}^{k}-\sum_{n:(n, i) \in \mathcal{A}} r_{n, i}^{k}= \begin{cases}1 & \text { if } i=o(k) \\
-1 & \text { if } i=d(k) \\
0 & \text { otherwise }\end{cases}
\end{aligned}
$$

\section{Methodology}

We describe the metrics, settings, and baselines. Throughout the study, we use the Cooja/MSPSim simulator [8]. MSPSim emulates the MSP430 MCU and the CC2420 radio chip at instruction level, providing time-accurate executions.

Comparison baselines. Our primary goal is to compare the DPF protocols against the standard OMNI stack, both described in Section IV. However, our DPF variants all rely on a routing tree built atop omni-directional communication, and disregard the ability to build and maintain the tree using directional communication. This would exploit the increased range of ESD antennas, reducing the route stretch and improving reliability and lifetime. The problem is open [7], and

\begin{tabular}{|l|c|c|}
\hline Dimension & Values & Unit metric \\
\hline \hline Packet generation rate & $1 \ldots \mathbf{3} \ldots 10$ & packets/min \\
\hline Number of nodes & $100 \ldots \mathbf{3 0 0} \ldots 500$ & nodes \\
\hline Network density & $4 \ldots \mathbf{1 2} \ldots 20$ & nodes/unit square \\
& $(2.88) \ldots(\mathbf{8 . 6 4}) \ldots(14.4)$ & (neighbors in omni) \\
\hline
\end{tabular}

TABLE I

System dimensions. Default Values are in boldface.

it is difficult to establish a term of comparison. Therefore, we opt for independently evaluating the trade-offs along two components: packet forwarding and control traffic.

As for the former, we compare the protocols against a DPF variant called DIRTREE, operating on the optimal routing topology found in Section V. The latter includes information on the direction to use to transmit to a parent, and for the parent what direction to use to send acknowledgments. Simulations with DIRTREE execute with this routing information hard-wired in the nodes. To make the executions of DIRTREE comparable with the others-which also account for control traffic-we record the control traffic generated with OMNI, and re-play it during DIRTREE simulations.

However, a real protocol exploiting directional communications would hopefully improve over the OMNI baseline. For this reason, we also compare against the traffic generated solely by data forwarding. This assesses the efficiency of the routing topology alone, no matter how it is obtained. In a sense, these two extremes-an optimal directional tree with the same traffic as the standard omni-directional stack, and one built "for free"-define the margin of improvement available to clean-slate protocols based on directional communication.

Metrics. We consider two key performance metrics commonly used in studying WSN protocols [13]: i) packet delivery at the sink, defined as the fraction of application packets successfully received at the sink over those sent; and ii) radio duty cycle, computed as the fraction of time a node keeps the radio on over the experiment duration. To determine packet delivery at the sink, we embed sequence numbers within data packets. We measure the radio duty cycle in software, using Contiki's power profiler. For each setting and protocol analyzed, we compute these metrics based on at least 50 statistically independent runs and report network-wide averages, along with their standard deviation. Simulations lasts until the system reaches stable conditions, a situation we verify by imposing that all per-node standard deviations for all metrics are below $5 \%$ of the average value.

Settings. We explore different system dimensions, illustrated in Table I with their default values. The packet generation rates reflect the settings of real-world WSN deployments [11]. Instead, the number of nodes involved purposely exceeds the size of real installations, yielding challenging conditions for the protocols. As for network density, Table I reports the number of nodes per unit square, $100 \mathrm{~m}$ in our experiments. The actual network density, however, is a function of the antenna employed. To relate our values with the existing literature, the table also reports the average number of neighbors when using SPIDA in omni-directional mode, which is in line with existing deployments [11]. 
We use 80-byte packets for all experiments, again inspired by real deployments [11]. We start measuring after 10 minutes of simulated time to let the protocols converge to an initial routing topology. In ContikiMAC, we set the periodic wakeup interval to $8 \mathrm{~Hz}$. We use the same default settings for all protocols' parameters.

We employ our empirical link-layer model, described in Section III. For consistency of results, we simulate omnidirectional transmissions still with our model as opposed to Cooja's built-in models, by isolating all parasitic elements of SPIDA. Note that Cooja does not model capture effects [16], and is thus very pessimistic when simulating interferences between different transmitters and hidden terminals. For each simulation run, we generate the network topologies randomly using Cooja's default mechanisms. The antenna orientation in the $2 \mathrm{D}$ plane and the placement of the data sink are also randomly chosen and different for every run.

\section{EVALUATION}

We assess the benefits of DPF using the protocols in Section IV and discuss where further improvements are available based on deeper modifications to the protocols or to the antenna design.

\section{A. Assessing Directional Packet Forwarding}

We evaluate our changes to Collect to enable DPF. Our results reveal that: $i$ ) DPF provides significant gains in packet delivery and radio duty-cycle compared to OMNI; ii) these gains apply to a variety of settings, and are mainly due to the ability of reaching the parent with higher signal strength and, for SMART and NARROW, to better spatial utilization of the channel; and iii) DIRTREE, which additionally uses optimal routing topologies based on directional transmission, does not provide significant additional gains, except when the size of the network grows.

Effect of network density. Figure 5 depicts the performance of the protocols we test against the number of nodes per unit square. The DPF solutions show improved delivery at the sink, as shown in Figure 5(a). By steering the radiated power towards the parent, both SMART and NARROW generate less contention on the channel, yielding fewer packets lost in collisions. The benefits become more evident as more nodes are potentially in reach. NARROw performs slightly worse than other directional solutions as it takes more time to find another working direction upon changes in the link quality.

The good performance of BLIND in Figure 5(a) is somewhat unexpected, as it sweeps all antenna directions for every packet, supposedly causing more collisions-and therefore packet losses-than SMART and NARROW. Looking at the simulation logs, we verify that BLIND causes about $27 \%$ more collisions on average than OMNI. However, most of these collisions happen when using directions other than the one best for a parent, and are thus immaterial. Thus, BLIND still ensures that the best direction to send data is eventually used.

In Figure 5(a) we also note that DIRTREE performs comparably to other directional solutions. In these scenarios and with

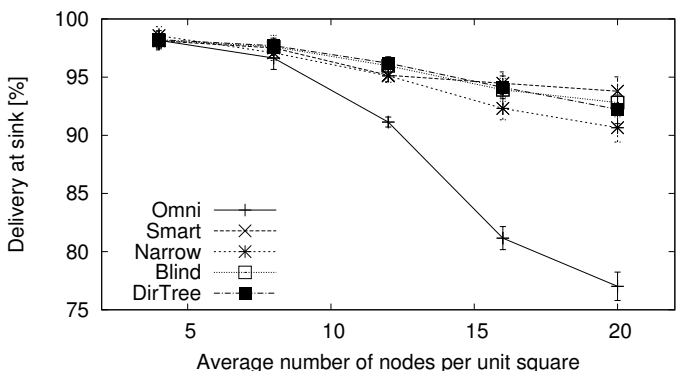

(a) Average packet delivery: All DPF solutions improve packet delivery for higher node densities.

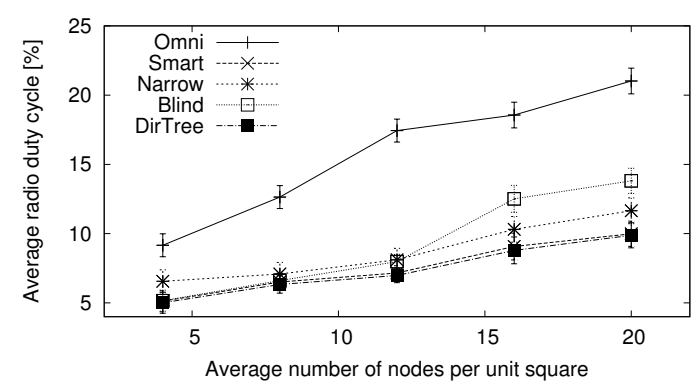

(b) Average radio duty-cycle. DPF also reduces the radio duty cycle, saving energy.

Fig. 5. Performance against varying network density.

the protocol stack we consider, leveraging routes found with directional transmissions does not provide additional gains. We verified from the simulation logs that with increasing network density it becomes equally likely for parents found beyond the omni-directional range to be affected by collisions. This neutralizes the benefits of the increased communication range when finding parents, and even causes DIRTREE to perform slightly worse than other directional solutions at times.

Figure 5(b) shows the radio duty cycle in the same settings. The gains in packet delivery using DPF yield savings in the energy spent for delivering packets. Indeed, improved reliability at packet level entails fewer re-transmissions. Among the protocols in Section IV, BLIND exhibits the worst performance. BLIND always sweeps all directions for every packet, and thus it necessarily keeps the radio on slightly longer.

Effect of network traffic. The protocols' performance against increasing packet generation rates, reported in Figure 6, confirms the discussion above. Indeed, injecting more packets into the network ultimately causes channel contention similarly to increasing network density. Packet delivery improves with directional transmissions compared to OMNI as the packet generation rate increases, as shown in Figure 6(a). DIRTREE, on the other hand, provides again only limited additional gains, due to collisions occurring in the region where omnidirectional transmissions do not reach.

DPF delivers more packets with smaller radio duty cycles, as reported in Figure 6(b), with BLIND performing the worst for the reasons explained previously. It attains performance similar to or better than other directional solutions only at low traffic or in sparse networks, where not much contention exists. Here, BLIND leverages the fact that the best direction for a parent is always eventually used. In the same chart, DIRTREE 


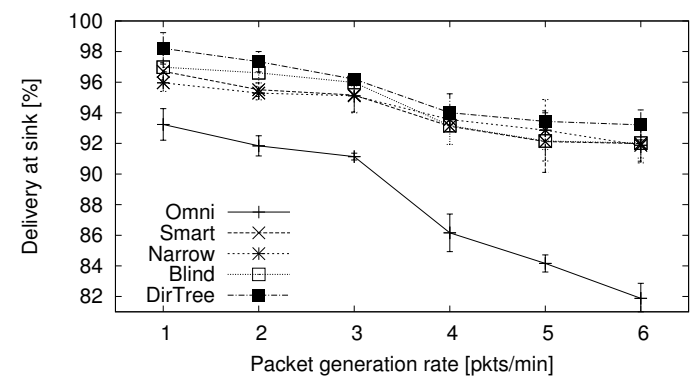

(a) Average packet delivery. DPF sustains high packet delivery for high traffic loads.

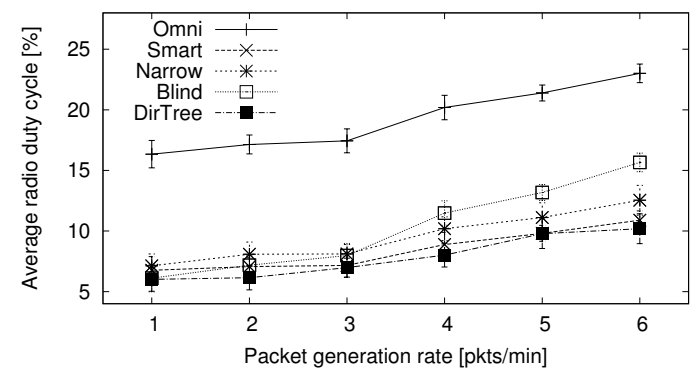

(b) Average radio duty-cycle. DPF improves the radio duty-cycle at different traffic loads.

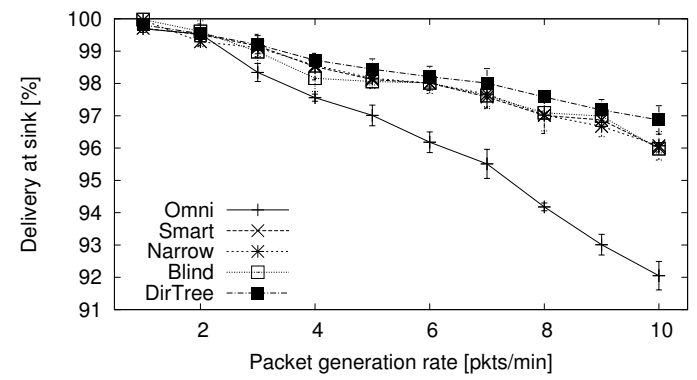

(c) Average packet delivery - no radio duty cycle. DPF sustains high packet delivery even at very high traffic loads.

Fig. 6. Performance against varying packet rate. Note the different scales in the packet delivery charts.

is indeed the lower bound, but again it improves over SMART only by $1.7 \%$ on average.

Figure 6(a) and 6(b) stop at 6 packets/min because we find all protocols unable to sustain higher traffic loads. Indeed, ContikiMAC reduces the bandwidth because of radio duty cycling: at 7 packets/min, all protocols deliver less than $40 \%$ of packets to the sink, rendering the results meaningless. To study higher traffic loads, we temporarily disable radio duty cycling in ContikiMAC, which leads to the performance in Figure 6(c). The trends in the chart mirror Figure 6(a), but on a different $\mathrm{X}$ - and Y-scale, demonstrating that the MAC protocol was the limiting factor. The conclusions on the influence of DPF therefore still apply, including the limited gains of DIRTREE. Effect of network size. Figure 7 shows the performance with increasing scale. As before, all other dimensions-network density and packet generation rate in this case-remain unaltered. Unlike with these two dimensions, however, increasing scale shows some advantages for DIRTREE besides the gains of DPF: Figure 7(a) shows DIRTREE increasingly delivering more packets as the network size grows. It does so with

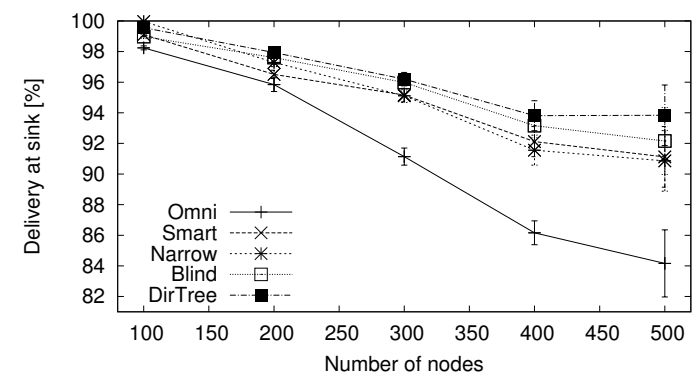

(a) Average packet delivery. DPF sustains high packet delivery for large networks. DIRTREE shows additional improvements.

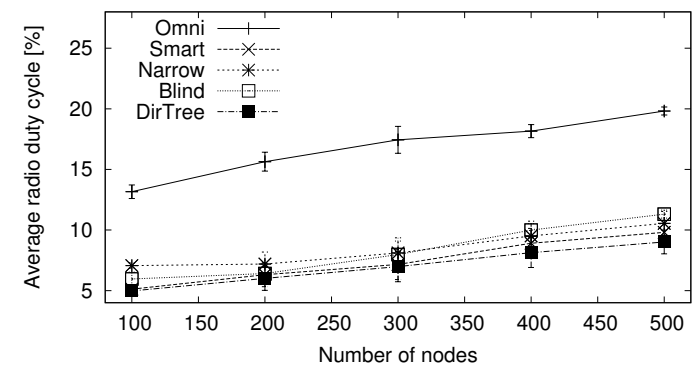

(b) Average radio duty-cycle. DPF keeps the radio duty-cycle low for large networks.

Fig. 7. Performance against varying number of nodes.

progressively better performance in radio duty-cycling, as illustrated in Figure 7(b). This is because the gains of DIRTREE are essentially "localized" in the single neighborhoods, and increasing the network size replicates such gains across more neighborhoods. Differently, network density and packet generation rate mainly affect channel contention, a situation where building routes based on directional transmissions does not help more than using DPF alone.

The results in this section exemplify the readily available benefits of employing ESD antennas in WSNs. The question is then whether further gains are available with deeper modifications to the stack or different antenna designs. We investigate the problem in the following two sections.

\section{B. The Cost of Control Traffic}

As we mention in Section VI, DIRTREE runs by re-playing the control traffic of OMNI. This traffic is intimately tied to the specific stack employed, which uses ETX as link quality metric and CSMA to access the radio medium. Next, we factor out the control traffic to understand the potential improvements with different routing strategies.

We re-run the simulation scenarios of Section VII-A with two additional baselines: $i$ ) DIRTREE-NOCONTROL works the same as DIRTREE without re-playing control traffic; and ii) OMNI-NoControl works the same as OMNI but also spares the control traffic by relying on an optimal routing topology computed similarly to DIRTREE, but based solely on omni-directional transmissions. These schemes provide a means to measure the effectiveness of the packet forwarding functionality alone, without any control overhead. Therefore, they represent a theoretical upper bound for any solution using directional and omni-directional transmissions, respectively. 


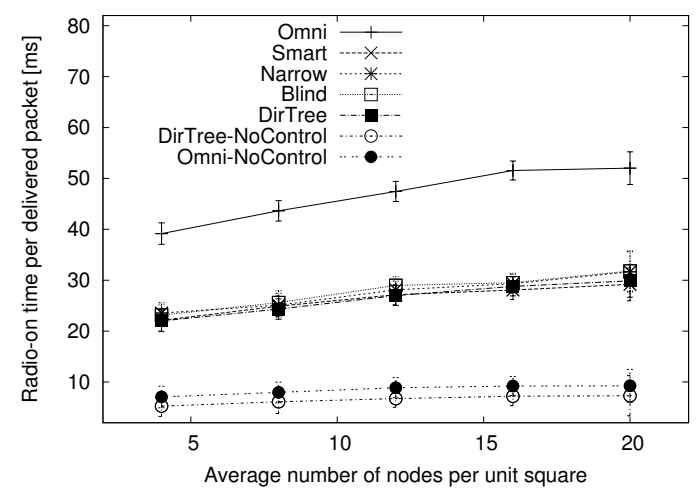

Fig. 8. Radio-on time per delivered packet against network density. DPF halves radio-on time. Without control traffic, radio-on time is further reduced.

We measure the average radio-on time spent per delivered packet-representing the unit cost of delivering a packet to the sink- to provide a concise indication on the effectiveness of a given schema. In most cases, both DIRTREE-NOCONTROL and OMNI-NOCONTROL deliver close to $100 \%$ of packets at the sink: without control traffic, the few remaining collisions rarely cause packet losses.

Results. Figure 8 shows the trends w.r.t. varying network density, although the following observations also apply w.r.t. varying packet generation rates and number of nodes.

The chart shows that DPF saves about half of the control overhead in OMNI: DPF solutions lie halfway between OMNI and OMNI-NOCONTROL. This complements the results of Section VII-A, further evidencing that simple modifications to existing protocols already enable significant improvements.

We also observe a significant gap between DPF solutions and DirTree-NoControl, roughly as large as the one between the former and OMNI. The overhead here is entirely due to the way routes are built and maintained-absent in DirTree-NoConTroL-based on ETX and broadcast beaconing in our case. This is the mainstream technique with omni-directional transmissions, but the extensive use of broadcast poorly matches the operation of directional antennas, for which more efficient protocols may be designed. The gap quantifies the margin of improvement available.

Finally, we note that DIRTREE-NOCONTROL performs about 30\% better than OMNI-NOCONTROL, which corresponds to the reduction in overall route stretch enabled by the larger communication range of SPIDA when in directional mode (Figure 3). Indeed, in our simulations the average tree depth for DirTree-NoControl is about one third smaller than OMNI-NOCONTROL. We further discuss this aspect next, as this is where the operation of network protocols meets the antenna characteristics, and one may find opportunities for different antenna designs.

\section{Opportunities for Antenna Designers}

Intuitively, the effect of reducing the route stretch-key to many of the improvements discussed thus far-should be amplified by an antenna design that further increases the communication range. The open question, however, is to what extent this impacts the overall performance.

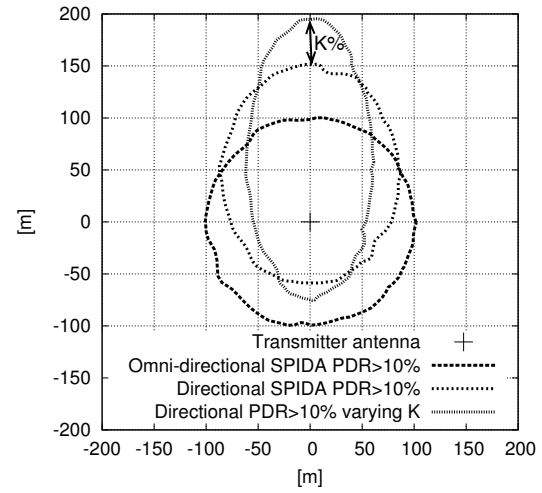

Fig. 9. Region of space with $P D R>10 \%$, depending on $K$.

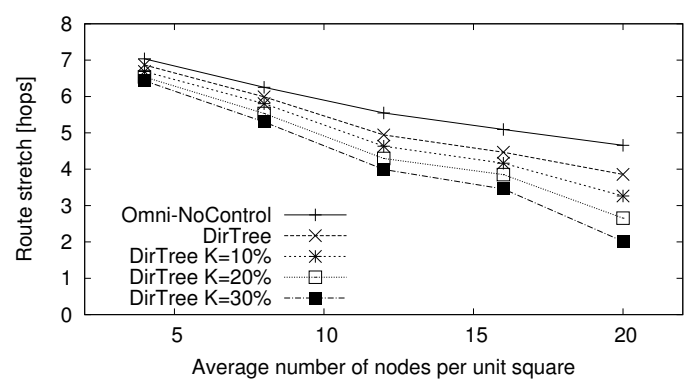

Fig. 10. The route stretch decreases when $K$ and network density increase.

To answer this, we artificially adapt our empirical link-layer model as shown in Figure 9. We change the shape of the main lobe to increase the communication range at the expense of spatial coverage perpendicular to the direction of maximum gain. In particular, we keep constant the area covered by the main lobe-in a sense proportional to the energy invested in transmission-but redistribute the $P D R$ information to increase the maximum communication range by $K \%$. Details on the analytical derivation are in an extended report [17]. We test values up to $K=30 \%$, in line with the technological limits of practical directional antenna designs [22].

Figure 10 shows how the route stretch varies w.r.t. network density, for the optimal off-line topology used in DirTree and DirTree-NoControl. For comparison, the omni-directional case shown is also based on the optimal routing topology used in OMNI-NOCONTROL. We consider only optimal schemes to factor out possible inefficiencies of the specific protocol in building the routing topology. As expected, the chart shows that increased network density causes a decrease in route stretch, as seen in all curves. As more nodes are in reach, shorter routes become possible. However, directional antennas amplify this effect. At the maximum density we consider, DIRTREE with the original SPIDA yields routes $30 \%$ shorter; when $K=30 \%$, routes are $60 \%$ shorter.

To evaluate the impact of reduced route stretch, we use our modified link-layer model to re-run the simulations in Section VII-A. We consider only SMART among the DPF solutions of Section IV, as it represents the best trade-off between packet delivery and radio duty-cycle.

Results. Figure 11(a) shows that antennas with larger communication ranges improve packet delivery only at a specific network density. The latter is the "sweet spot" where the 


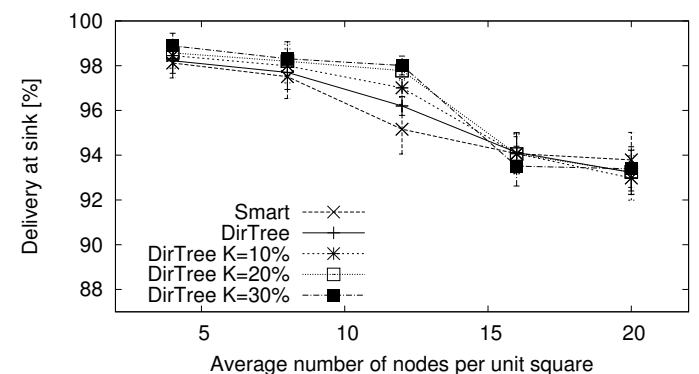

(a) Average packet delivery. Packet delivery improves only at a specific network density with increasing $K$.

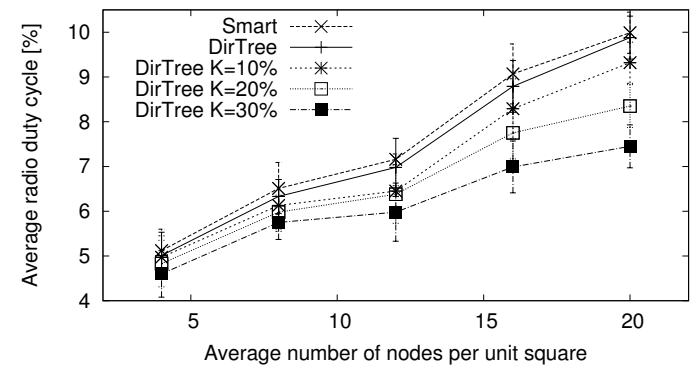

(b) Average radio duty-cycle. The radio duty-cycle decreases for increasing $K$ in all settings, saving energy.

Fig. 11. Performance of antenna designs against varying network density.

benefits to route stretch of directional transmissions are not countered by an excessive network density. When the network is too sparse, the increased communication range does not make a difference; there are few nodes to choose from, and routes are almost identical regardless of $K$. When the network is too dense, collisions dominate performance, annihilating the benefits of a reduced route stretch.

On the other hand, the radio duty-cycle improves in all settings, as shown in Figure 11(b). In sparse networks, the savings are enabled by the reduced need for retransmissions, given that the route topology is almost identical in all cases. Indeed, increasing $K$ yields a stronger signal and therefore a better $P D R$ at the parent. We expect this behavior to manifest more prominently in real networks, due to the capture effect [16]. In denser networks, routes are shorter and therefore fewer overall transmissions are necessary.

Similar trends and considerations hold for the results obtained by varying packet generation rates, as in Section VII-A. When varying the number of nodes, instead, the gains "multiply" across more nodes, as larger communication ranges allow to cover the network in fewer hops: DIRTREE with $K=30 \%$ almost halves the radio duty-cycle compared to SMART.

These quantitative results offer antenna designers an indication of the margin of improvement available, motivating realworld designs similar to the one we artificially create.

\section{CONCLUSIONS}

The benefits of directional transmissions are vastly demonstrated on cellular and ad-hoc networks. The WSNs literature lacks a comparable understanding of the benefits at hand, due to the lack of comprehensive evaluations based on practical assumptions. Based on an empirical link-layer model from a real-world ESD antenna, we showed that minimal changes to an existing protocol enable significant improvements. Our results also suggest that clean-state protocol designs that focus on the control rather than the data plane may bring similar additional gains, and indicate further room for improvements with different antenna designs.

Acknowledgements. This work was carried out within WISENET (Uppsala VINN Excellence Center for Wireless Sensor Networks) funded by VINNOVA. We also wish to thank Martin Nilsson, who designed the SPIDA antenna, as well as Fredrick Österlind and Antonio Quartulli for early contributions to this work.

\section{REFERENCES}

[1] M. Arslan et al. Design and implementation of an integrated beamformer and uplink scheduler for OFDMA femtocells. In MobiHOC, 2012.

[2] N. Baccour et al. Radio link quality estimation in wireless sensor networks: a survey. ACM Trans. on Sensor Networks, 8(4), 2012.

[3] M. Buettner et al. X-MAC: a short preamble MAC protocol for dutycycled wireless sensor networks. In SENSYS, 2006.

[4] A. Cerpa et al. Statistical model of lossy links in wireless sensor networks. In IPSN, 2005.

[5] R. Choudhury et al. Beamnet: An ad hoc network testbed using beamforming antennas. In Vehicular Technology Conference, 2005.

[6] D. D. Couto et al. A high-throughput path metric for multi-hop wireless routing. Wirel. Netw., 11(4), 2005.

[7] H. Dai et al. An overview of using directional antennas in wireless networks. Communication Systems, Nov. 2011.

[8] J. Eriksson et al. COOJA/MSPSim: Interoperability testing for wireless sensor networks. In SIMUTools, 2009.

[9] E. Felemban et al. Samac: A cross-layer communication protocol for sensor networks with sectored antennas. IEEE Transactions on Mobile Computing, 9(8), 2010.

[10] E. Felemban et al. Sand: Sectored-antenna neighbor discovery protocol for wireless networks. In SECON, 2010.

[11] E. Gaura et al. Wireless Sensor Networks: Deployments and Design Frameworks. Springer, 2010.

[12] G. Giorgetti et al. Exploiting low-cost directional antennas in $2.4 \mathrm{GHz}$ IEEE 802.15.4 wireless sensor networks. In European Conf. on Wireless Technologies, 2007.

[13] O. Gnawali et al. Collection tree protocol. In SENSYS, 2009.

[14] M. Keller, J. Beutel, and L. Thiele. How was your journey? Uncovering routing dynamics in deployed sensor networks with multi-hop network tomography. In SENSYS, 2012.

[15] J. Ko et al. Beyond interoperability: Pushing the performance of sensornet IP stacks. In SENSYS, 2011.

[16] J. Lu and K. Whitehouse. Flash flooding: Exploiting the capture effect for rapid flooding in wireless sensor networks. In INFOCOM, 2009.

[17] L. Mottola et al. Electronically-switched directional antennas for lowpower wireless networks: A prototype-driven evaluation. Technical report, Swedish Institute of Computer Science, 2012.

[18] L. Mottola and G. P. Picco. Muster: Adaptive energy-aware multisink routing in wireless sensor networks. IEEE Transactions on Mobile Computing, 10(12), 2011.

[19] M. Nilsson. Directional antennas for wireless sensor networks. In Scandinavian Workshop on Wireless Adhoc Networks, 2009.

[20] E. Öström, L. Mottola, and T. Voigt. Evaluation of an electronically switched directional antenna for real-world low-power wireless networks. In Real-World Wireless Sensor Networks (REALWSN), 2010.

[21] K. Srinivasan et al. The $\beta$-factor: measuring wireless link burstiness. In SENSYS, 2008.

[22] D. Thiel and S. Smith. Switched parasitic antennas for cellular communications. Artec House, London, 2002.

[23] S. Vasudevan, J. Kurose, and D. Towsley. On neighbor discovery in wireless networks with directional antennas. In INFOCOM, 2005.

[24] F. Viani et al. Exploitation of parasitic smart antennas in wireless sensor networks. Electromagnetic Waves and Applications, 24(7), 2010.

[25] B. Y. Wu and K.-M. Chao. Spanning Trees and Optimization Problems. Chapman \& Hall, 2004. 